\title{
Pitch-Angle Dependent Perpendicular Diffusion of Energetic Particles Interacting With Magnetic Turbulence
}

\author{
Gang Qin $^{1} \&$ Andreas Shalchi ${ }^{2}$ \\ ${ }^{1}$ State Key Laboratory of Space Weather, Center for Space Science and Applied Research, Chinese Academy of \\ Sciences, Beijing, China \\ 2 Department of Physics and Astronomy, University of Manitoba, Winnipeg, Manitoba, Canada \\ Correspondence: Andreas Shalchi, Department of Physics and Astronomy, University of Manitoba, Winnipeg, \\ Manitoba R3T 2N2, Canada. E-mail: andreasm4@yahoo.com
}

Received: August 17, 2013 Accepted: September 2, 2013 Online Published: December 19, 2013

doi:10.5539/apr.v6n1p1 URL: http://dx.doi.org/10.5539/apr.v6n1p1

\begin{abstract}
We employ a test-particle code to explore diffusion of energetic particles interacting with the solar wind plasma. The first time we investigate the pitch-angle dependence of perpendicular diffusion for different parameter regimes. These results are important for numerical solutions of the pitch-angle dependent Cosmic Ray Fokker-Planck equation. We compare our finding also with predictions made by analytical theory.
\end{abstract}

Keywords: diffusion, turbulence, cosmic rays

\section{Introduction}

In the present article we explore the interaction of energetic particles such as cosmic rays with the solar wind plasma by using numerical simulations. The propagation and acceleration of such particles is usually modeled by solving the cosmic ray transport equation (Parker, 1965). A fundamental quantity which enters the diffusive transport equation is the diffusion tensor describing the stochastic motion of the particles. The latter tensor contains, in the general case, the diffusion coefficient along the mean magnetic field $\kappa_{\|}$, the perpendicular diffusion coefficient $\kappa_{\perp}$, and the drift coefficient $\kappa_{A}$. Here we assumed axi-symmetry with respect to the mean field $\vec{B}_{0}$. Usually the different transport coefficients depend on turbulence parameters and the particle rigidity/energy.

A more fundamental description can be achieved if the diffusive transport equation discussed above is replaced by the so-called cosmic ray Fokker-Planck equation (see, e.g., Schlickeiser, 2002). In this case the particle intensities depend also on the pitch-angle cosine defined as $\mu=v_{\|} / v$ where $v$ is the particle speed and $v_{\|}$is the parallel component of the particle velocity. If we describe particle transport by using the Fokker-Planck equation instead of Parker's diffusion equation, all parameters in the latter equation depend on $\mu$. Instead of the parallel diffusion coefficient $\kappa_{\|}$the important quantity is now the pitch-angle Fokker-Planck coefficient $D_{\mu \mu}(\mu)$ and instead of the perpendicular spatial diffusion coefficient $\kappa_{\perp}$ we have to use the Fokker-Planck coefficient of perpendicular diffusion $D_{\perp}(\mu)$. The spatial diffusion coefficients are related to the pitch-angle dependent quantities via the relations (see, e.g., Schlickeiser, 2002)

and

$$
\kappa_{\|}=\frac{v^{2}}{8} \int_{-1}^{+1} d \mu \frac{\left(1-\mu^{2}\right)^{2}}{D_{\mu \mu}(\mu)}
$$

$$
\kappa_{\perp}=\frac{1}{2} \int_{-1}^{+1} d \mu D_{\perp}(\mu) .
$$

In which parameters one is interested in depends on whether the Fokker-Planck equation or the pitch-angle averaged cosmic ray transport equation has to be solved.

In test-particle simulations one simulates the magnetic field structure by employing certain turbulence models (e.g., isotropic turbulence, two-component turbulence, three-dimensional anisotropic turbulence) and then the NewtonLorentz Equation is solved numerically for a high amount of test-particles. From these particle orbits one can obtain the diffusion parameters $\kappa_{\|}$and $\kappa_{\perp}$. This procedure can be seen as standard approach and it was performed 
by numerous authors (see, e.g., Michałek \& Ostrowski, 1996; Mace et al., 2000; Qin et al., 2002a, 2002b; Pommois et al., 2005; Zimbardo et al., 2006; Pommois et al., 2007; Ruffolo et al., 2008; Tautz, 2010; Qin \& Shalchi, 2012; Tautz \& Shalchi, 2013). However, one can also be interested in the pitch-angle dependence of the different transport parameters. In particular one could try to compute the transport parameters $D_{\mu \mu}(\mu)$ and $D_{\perp}(\mu)$ from computer simulations. In Qin and Shalchi (2009) we started to explore the pitch-angle dependence of $D_{\mu \mu}(\mu)$. It is the purpose of the present paper to complement this previous work to explore the pitch-angle dependence of perpendicular diffusion.

To obtain pitch-angle dependent transport parameters is important to develop and test analytical theories for perpendicular diffusion and pitch-angle scattering (see, e.g., Shalchi 2009 for a review) but also for different astrophysical applications. For example, Florinski et al. (2008) and Zuo et al. (2011) studied the termination shock acceleration of pickup ions with the Fokker-Planck equation, they were able to discuss in detail different observational features of shock acceleration. In addition, Danos et al. (2013) solved the two-dimensional Fokker-Planck equation numerically to investigate the effect of adiabatic focusing on the motion of energetic particles. One of the parameters which enters such numerical work is the pitch-angle scattering coefficient $D_{\mu \mu}(\mu)$. In principle such investigations can be extended to take into account perpendicular diffusion described by the parameter $D_{\perp}(\mu)$.

Furthermore, Qin et al. (2006) studied the effect of pitch-angle dependent adiabatic cooling on the Solar Energetic Particles (SEPs) by numerically solving the Fokker-Planck equation, with the Markov stochastic process method (Zhang, 1999). In addition, by solving SEPs transport equation including pitch-angle independent perpendicular diffusion, Qin et al. (2011) showed that perpendicular diffusion can be used to approximately explain the Wind spacecraft observation of counterstreaming particle beams with a deep depression of flux at $\mu=0$ during the beginning of the event, which is usually explained by a reflecting boundary at some distance outside of 1 AU (Tan et al., 2009). It is possible to improve the modeling results of Qin et al. (2011) to agree with observations better by including a pitch-angle dependent perpendicular diffusion coefficient in the Fokker-Planck equation.

The paper is organized as follows: in Section 2, we discuss models for solar wind turbulence. Such models enter numerical as well as analytical work about test particle transport. In Section 3, we briefly discuss analytical results obtained for the pitch-angle dependent perpendicular diffusion coefficient. In Section 4, we explain the test particle code and show our new results. Finally, Section 5 provides a short summary and conclusion.

\section{Models for Solar Wind Turbulence}

An important quantity entering analytical transport theories as well as computer simulations is the magnetic correlation tensor defined as

$$
P_{l m}(\vec{k})=\left\langle\delta B_{l}(\vec{k}) \delta B_{m}^{*}(\vec{k})\right\rangle .
$$

In the following we discuss models for the latter quantity which should be valid in the solar wind.

\subsection{Slab/2D Turbulence}

In the solar wind it is usually assumed that turbulence can be described as superposition of slab and two-dimensional modes (see, e.g., Matthaeus et al., 1990, Zank \& Matthaeus, 1993).

By definition we have for slab turbulence

$$
P_{l m}^{s l a b}(\vec{k})=g^{s l a b}\left(k_{\|}\right) \frac{\delta\left(k_{\perp}\right)}{k_{\perp}} \delta_{l m}
$$

where we have used the Kronecker Delta $\delta_{l m}$ with $l, m=x, y$. To satisfy the solenoidal constraint, we need to have $P_{l z}=P_{z m}=P_{z z}=0$. Here $g^{s l a b}\left(k_{\|}\right)$is the (one-dimensional) turbulence spectrum of the slab modes.

Another turbulence model is that of pure two-dimensional (2D) turbulence where we have by definition

$$
P_{l m}^{2 D}(\vec{k})=g^{2 D}\left(k_{\perp}\right) \frac{\delta\left(k_{\|}\right)}{k_{\perp}}\left[\delta_{l m}-\frac{k_{l} k_{m}}{k^{2}}\right]
$$

where we have employed the Dirac Delta $\delta(z)$. Furthermore, we used the two-dimensional turbulence spectrum $g^{2 D}\left(k_{\perp}\right)$ which will be discussed below.

In the present paper we employ a slab/2D composite model where we assume a superposition of slab and twodimensional modes. In this case the correlation tensor is given by $P_{l m}^{s l a b / 2 D}=P_{l m}^{s l a b}+P_{l m}^{2 D}$ where the two individual components are given by Equations (2) and (3), respectively. 


\subsection{The Shalchi \& Weinhorst Model Spectrum}

Shalchi and Weinhorst (2009) proposed the following form for the spectrum of the two-dimensional modes

$$
\begin{aligned}
g^{2 D}\left(k_{\perp}\right) & =\frac{2 D(s, q)}{\pi} \delta B_{2 D}^{2} l_{2 D} \\
& \times \frac{\left(k_{\perp} l_{2 D}\right)^{q}}{\left[1+\left(k_{\perp} l_{2 D}\right)^{2}\right]^{(s+q) / 2}} .
\end{aligned}
$$

This form of the spectrum is correctly normalized. Above we have used the function

$$
D(s, q)=\frac{\Gamma\left(\frac{s+q}{2}\right)}{2 \Gamma\left(\frac{s-1}{2}\right) \Gamma\left(\frac{q+1}{2}\right)}
$$

where $\Gamma(z)$ is the Gamma function. The parameters used in the spectrum are the inertial range spectral index $s$, the energy range spectral index $q$, and the bendover scale of the two-dimensional modes $l_{2 D}$. The latter length scale denotes the turnover from the energy range of the spectrum to the inertial range. $\delta B_{2 D}$ denotes the total magnetic field associated with the two-dimensional modes.

As discussed in Shalchi and Weinhorst (2009), a similar spectrum can also used for the slab modes. In the following we only discuss a flat spectrum (with $q=0$ ) if the slab model is considered. In this special case the model spectrum is given by

$$
\begin{aligned}
g^{\text {slab }}\left(k_{\|}\right) & =\frac{C(s)}{2 \pi} \delta B_{\text {slab }}^{2} l_{\text {slab }} \\
& \times \frac{1}{\left[1+\left(k_{\|} l_{\text {slab }}\right)^{2}\right]^{s / 2}}
\end{aligned}
$$

with the same parameters as used for the two-dimensional modes (e.g., $l_{\text {slab }}$ is the slab bendover scale). Furthermore we have used

$$
C(s)=D(s, q=0)=\frac{\Gamma\left(\frac{s}{2}\right)}{2 \sqrt{\pi} \Gamma\left(\frac{s-1}{2}\right)} .
$$

This type of spectrum was used before in cosmic ray diffusion theory (see, e.g., Bieber et al., 1994; Teufel \& Schlickeiser 2003; Qin et al., 2002a, 2002b; Matthaeus et al., 2003; Ruffolo et al., 2004; Zank et al., 2004; Shalchi et al., 2004; Ruffolo et al., 2006).

The two spectra (4) and (6) are also in agreement with the forms discussed in Matthaeus et al. (2007).

\section{Analytical Considerations}

\subsection{The UNLT Theory}

Within the so-called Unifield-Non-Linear Transport (UNLT) theory developed by Shalchi (2010) the perpendicular diffusion coefficient is given by

$$
\kappa_{\perp}=\frac{a^{2} v^{2}}{B_{0}^{2}} \int d^{3} k P_{x x}(\vec{k}) \mathfrak{R}[T(\vec{k})]
$$

where $P_{x x}(\vec{k})$ is the $x x$-component of the magnetic correlation tensor. The parameter $a^{2}$ used here was introduced by Shalchi (2010) in the same way as originally in Matthaeus et al. (2003). The latter parameter is related to the probability that the particle is tied to a single magnetic field line.

The function $T(\vec{k})$ is given by

$$
T(\vec{k})=\frac{1}{2} \int_{-1}^{+1} d \mu \mu S(\mu, \vec{k})
$$

where $S(\mu, \vec{k})$ is the solution of the following ordinary differential equation

$$
-\mu=i v \mu k_{\|} S+\frac{\partial}{\partial \mu}\left[D_{\mu \mu} \frac{\partial S}{\partial \mu}\right]-k_{\perp}^{2} D_{\perp} S .
$$


Since the relation between the pitch-angle independent diffusion coefficient $\kappa_{\perp}$ and the pitch-angle dependent Fokker-Planck coefficient $D_{\perp}$ is given by

$$
\kappa_{\perp}=\frac{1}{2} \int_{-1}^{+1} d \mu D_{\perp}(\mu)
$$

we find from Equation (8)

$$
D_{\perp}(\mu)=\frac{a^{2} v^{2}}{B_{0}^{2}} \mu \int d^{3} k P_{x x}(\vec{k}) \Re[S(\mu, \vec{k})]
$$

where the function $S(\mu, \vec{k})$ is still obtained by solving Equation (10).

\subsection{Two-Dimensional Turbulence}

Above we have argued that the two-component model is an appropriate model for solar wind turbulence. However, there is no direct contribution to the perpendicular diffusion coefficient from the slab modes. The latter effect is sometimes called the Shalchi slab hypothesis (see Ruffolo et al., 2012) but it can easily derived directly from the UNLT theory (see, e.g., Shalchi, 2010). Therefore, only the two-dimensional modes contribute explicitly to the total perpendicular diffusion coefficient.

In the following we evaluate Equations (10) and (12) for the two-dimensional correlation tensor (3). For this specific turbulence model the Fokker-Planck coefficient of perpendicular diffusion becomes

$$
D_{\perp}(\mu)=\pi \frac{a^{2} v^{2}}{B_{0}^{2}} \mu \int_{0}^{\infty} d k_{\perp} g^{2 D}\left(k_{\perp}\right) S\left(\mu, k_{\|}=0\right)
$$

where $S$ is now provided by the solution of

$$
-\mu=\frac{\partial}{\partial \mu}\left[D_{\mu \mu} \frac{\partial S}{\partial \mu}\right]-k_{\perp}^{2} D_{\perp} S .
$$

The solution of the latter equation is difficult (see discussion in Shalchi, 2010; Lerche \& Tautz, 2011a, 2011b; Shalchi, 2011b). In the following we discuss two asymptotic solutions of (14).

\subsection{Dominant Perpendicular Diffusion}

If the perpendicular diffusion term in (14) is dominant we find the following equation

$$
-\mu=-k_{\perp}^{2} D_{\perp} S
$$

which has the solution

$$
S=\frac{\mu}{k_{\perp}^{2} D_{\perp}}
$$

and the Fokker-Planck coefficient (13) becomes

$$
D_{\perp}^{2}=\pi \frac{a^{2} v^{2}}{B_{0}^{2}} \mu^{2} \int_{0}^{\infty} d k_{\perp} g^{2 D}\left(k_{\perp}\right) k_{\perp}^{-2} .
$$

According to Equation (11) of Shalchi (2011a), the latter integral corresponds to the square of the field line diffusion coefficient for pure two-dimensional turbulence (see also Matthaeus et al., 1995, 2007)

$$
\kappa_{F L}^{2}=\frac{\pi}{B_{0}^{2}} \int_{0}^{\infty} d k_{\perp} k_{\perp}^{-2} g^{2 D}\left(k_{\perp}\right) .
$$

Therefore, we find in this limit

$$
D_{\perp}(\mu)=a v|\mu| \kappa_{F L}
$$

which is, for $a=1$, the well-known Field Line Randon Walk (FLRW) limit originally discussed in the pioneering work of Jokipii (1966). In this case the Fokker-Planck coefficient scales like $D_{\perp} \sim|\mu|$.

As shown in Shalchi and Weinhorst (2009), the field line diffusion coefficient for $q>1$ is given by

$$
\kappa_{F L}=\sqrt{\frac{s-1}{2(q-1)}} l_{2 D} \frac{\delta B_{2 D}}{B_{0}} .
$$


The pitch-angle independent perpendicular diffusion coefficient $\kappa_{\perp}$ can easily derived from (19) by pitch-angle averaging the latter equation. We find in this case

$$
\kappa_{\perp}=\frac{a v}{2} \kappa_{F L} .
$$

The latter formula is well-known in diffusion theory and was derived before from the UNLT theory (see, e.g., Shalchi, 2010). Below we focus on the pitch-angle dependent result (19).

\subsection{Dominant Pitch-Angle Scattering}

If the pitch-angle scattering term in (14) is dominant we find the following differential equation

$$
-\mu=\frac{\partial}{\partial \mu}\left[D_{\mu \mu} \frac{\partial S}{\partial \mu}\right]
$$

In this case the function $S$ does not depend on the wavenumber $k_{\perp}$ and the Fokker-Planck coefficient (13) becomes

$$
D_{\perp}(\mu)=\pi \frac{a^{2} v^{2}}{B_{0}^{2}} \mu S(\mu) \int_{0}^{\infty} d k_{\perp} g^{2 D}\left(k_{\perp}\right) .
$$

With the normalization condition

$$
\int_{0}^{\infty} d k_{\perp} g^{2 D}\left(k_{\perp}\right)=\frac{\delta B_{2 D}^{2}}{2 \pi}
$$

this reads

$$
D_{\perp}(\mu)=\frac{a^{2} v^{2}}{2} \frac{\delta B_{2 D}^{2}}{B_{0}^{2}} \mu S(\mu)
$$

where $S(\mu)$ is still given by the solution of (22). If we integrate the latter equation once we find

$$
\frac{\partial S}{\partial \mu}=\frac{1-\mu^{2}}{2 D_{\mu \mu}}
$$

Obviously the latter equation can only be solved if the pitch-angle dependence of $D_{\mu \mu}$ is known.

Motivated by the simulations of Qin and Shalchi (2009) and section IV of the present paper, we employ the isotropic form

$$
D_{\mu \mu}=(1-\mu)^{2} D
$$

where $D$ is a pitch-angle independent constant. In this case Equation (26) can easily be solved by

$$
S(\mu)=S(\mu=0)+\frac{\mu}{2 D}
$$

Since we expect (due to symmetry considerations) $D_{\perp}$ to be an even function of $\mu$ we can set $S(\mu=0)=0$ and the Fokker-Planck coefficient of perpendicular diffusion (25) becomes

$$
D_{\perp}(\mu)=\frac{a^{2} v^{2} \mu^{2}}{4 D} \frac{\delta B_{2 D}^{2}}{B_{0}^{2}}
$$

If one is just interested in the pitch-angle independent perpendicular diffusion coefficient $\kappa_{\perp}$, one can $\mu$-average the latter result to obtain

$$
\kappa_{\perp}=\frac{a^{2} v^{2}}{12 D} \frac{\delta B_{2 D}^{2}}{B_{0}^{2}}=\frac{a^{2}}{2} \kappa_{\|} \frac{\delta B_{2 D}^{2}}{B_{0}^{2}}
$$

where we have used $6 D=v^{2} / \kappa_{\|}$(see, e.g., Shalchi, 2006). Formula (30) is well-known in diffusion theory and was derived before (see, e.g., Shalchi, 2013).

\section{Simulations}

In this work, we use numerical simulations to study pitch-angle dependent perpendicular diffusion coefficients. We use the same simulation code as that in Qin and Shalchi (2012), which was based on the previous code (see, e.g., Mace et al., 2000; Qin et al., 2002a, 2002b). In the code, we use a periodic box of size $10000 l_{\text {slab }}$ and 
$N_{z}=4194303$ points to generate the slab component of magnetic turbulence, as well as a periodic box of size $100 l_{\text {slab }} \times 100 l_{\text {slab }}$ and $N_{x} \times N_{y}=4096 \times 4096$ points to generate the two-dimensional component of magnetic turbulence. Here, we set the inertial range index $s=5 / 3$. In addition, we use a Fourier analysis to obtain magnetic turbulence in real space from that in $k$-space. With a fourth-order Runge-Kutta method with adaptive step size control of accuracy of $10^{-9}$, we solve the Newton-Lorentz equation to get the particle trajectories, from which the running diffusion coefficients are obtained.

Here, in order to get the pitch-angle dependent perpendicular diffusion coefficients, we have to choose the cases with lower pitch-angle diffusion but higher perpendicular diffusion so that there is a time scale during which perpendicular diffusion is obtained with the pitch-angle not changing too frequently. We do simulations for different values of ratios of particle gyro-radius to bendover scale of slab component, $R_{L} / l_{\text {slab }}$, and the ratio of bendover scales between two-dimensional component and slab component, $l_{2 D} / l_{\text {slab }}$. Note that we set turbulence level, $\delta B / B_{0}=0.1$, the ratio of two-dimensional component magnetic energy to the slab component magnetic energy, $\delta B_{2 D}^{2}: \delta B_{\text {slab }}^{2}=80: 20$, and the energy range index of the two-dimensional modes $q=1.5$ in all simulations. In addition, we fit the pitch-angle scattering coefficient $D_{\mu \mu}(\mu)$ to the isotropic form (27).

The simulation runs are summarized in Table 1 and shown in Figures 1-6. In bottom panels of Figures 1-6 we show the pitch-angle scattering coefficient $D_{\mu \mu}(\mu)$ from simulation results (dots) and fitting results with Equation (27) (dotted-lines). In addition, in top panels of Figures 1-6 we show the pitch-angle dependent perpendicular diffusion coefficients $D_{\perp}(\mu)$ from simulation results (dots) and theory of Equation (19) with $a^{2}=1 / 10$ (dotted lines) and $a^{2}=1 / 3$ (dashed lines). The horizontal axis, $\mu$, for simulation results, is obtained by averaging for particles throughout the simulations.

Table 1. The different test-particle simulations performed in the present paper. For all runs the inertial range spectral index is $s=5 / 3$, the energy range index of two-dimensional modes is $q=1.5$, the turbulence level is $\delta B / B_{0}=0.1$, and the ratio of two-dimensional component magnetic energy to the slab component magnetic energy is $\delta B_{2 D}^{2}: \delta B_{\text {slab }}^{2}=80: 20$

\begin{tabular}{cccc}
\hline Run & $l_{2 D} / l_{\text {slab }}$ & $R_{L} / l_{\text {slab }}$ & Figure \\
\hline 1 & 0.2 & 0.1 & 1 \\
2 & 0.2 & 1 & 2 \\
3 & 0.2 & 10 & 3 \\
4 & 0.2 & 40 & 4 \\
5 & 0.2 & 100 & 5 \\
6 & 0.5 & 100 & 6 \\
\hline
\end{tabular}

\subsection{Parameters and Results for Run I}

In Figure 1 we set the ratio of particle gyro-radius to the slab modes correlation scale $R_{L} / l_{\text {slab }}=0.1$ and the ratio of correlation scales between two-dimensional component and slab component, $l_{2 D} / l_{\text {slab }}=0.2$. In the top panel we show $D_{\perp}(\mu)$ from simulations with dots and that from Equation (19) with $a^{2}=1 / 10$ (dotted line) and $a^{2}=1 / 3$ (dashed line). It is shown that the simulation results of $D_{\perp}(\mu)$ agree well with Equation (19) with the $a^{2}=1 / 3$.

In the lower panel we show $D_{\mu \mu}(\mu)$ from simulations with dots. In addition, we show the fitting results of $D_{\mu \mu}$ to Equation (27) with dotted-line. In this particular case $D_{\mu \mu}(\mu)$ does not have an isotropic form as described by Equation (27). 


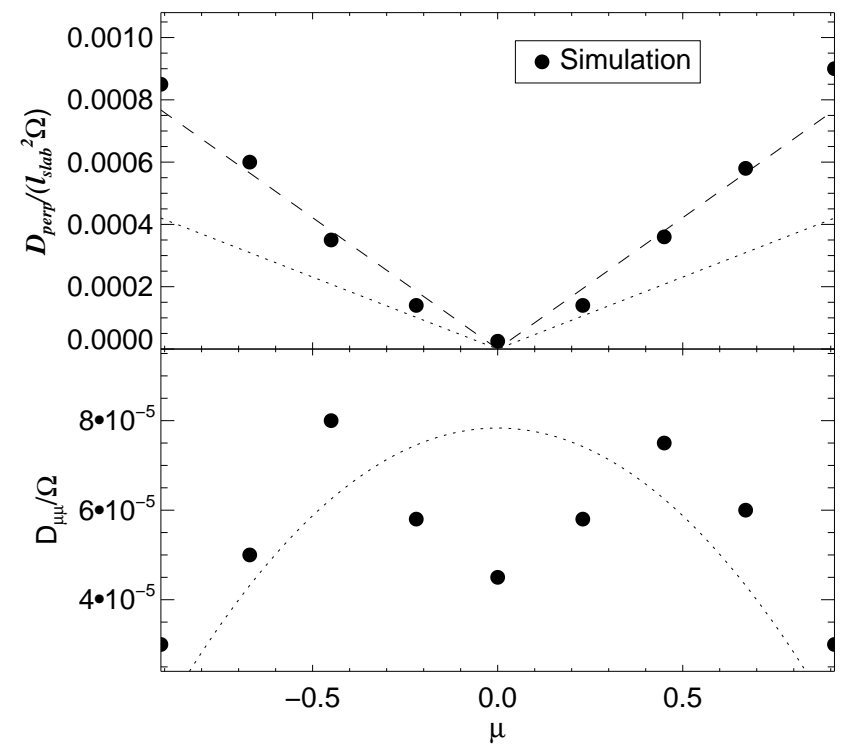

Figure 1. Top panel: The pitch-angle dependent perpendicular diffusion coefficient $D_{\perp}(\mu)$ from computer simulations (dots) compared with Equation (19) with $a^{2}=1 / 10$ (dotted line) and $a^{2}=1 / 3$ (dashed line). Bottom panel: The pitch-angle diffusion coefficient $D_{\mu \mu}(\mu)$ from the simulation (dots) compared with Equation (27) (dotted line). For the particle and turbulence parameters we used $R_{L} / l_{\text {slab }}=0.1$ and $l_{2 D} / l_{\text {slab }}=0.2$ to obtain these plots

\subsection{Parameters and Results for Run II}

Figure 2 is similar as Figure 1 except that we consider higher particle energies with $R_{L} / l_{\text {slab }}=1$. It is shown that the simulation results of $D_{\perp}(\mu)$ agree well with Equation (19) if we set $a^{2}=1 / 3$. In addition, the simulations provide an almost isotropic form for $D_{\mu \mu}(\mu)$ in agreement with Equation (27).

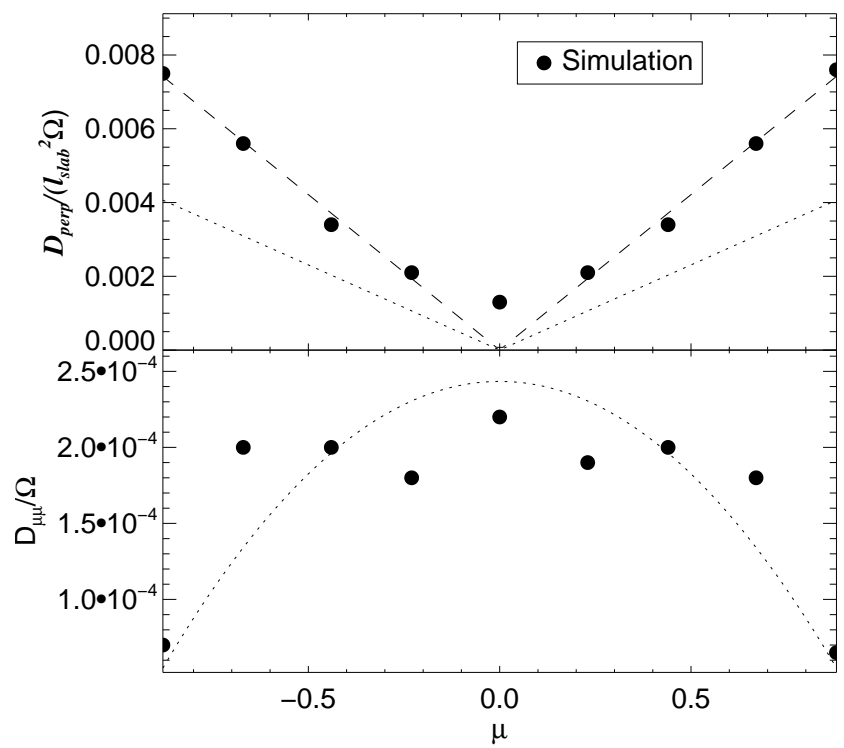

Figure 2. The same as Figure 1 except that $R_{L} / l_{\text {slab }}=1$ 


\subsection{Parameters and Results for Run III}

To obtain Figure 3 we have further increased the particle energy by setting $R_{L} / l_{\text {slab }}=10$. It is shown that when $|\mu|>0.1$ the simulation results of $D_{\perp}(\mu)$ agree well with Equation (19) with $a^{2}=1 / 3$. In addition, when $|\mu|>0.5$, the simulation results are smaller than the theoretical results with $a^{2}=1 / 3$. It suggests a smaller value of $a^{2}$. But when $|\mu|<0.1$, the simulation results of $D_{\perp}(\mu)$ is much larger than the theoretical results from Equation (19). For the case considered here we find an almost isotropic form for the parameter $D_{\mu \mu}(\mu)$ in agreement with Equation (27).

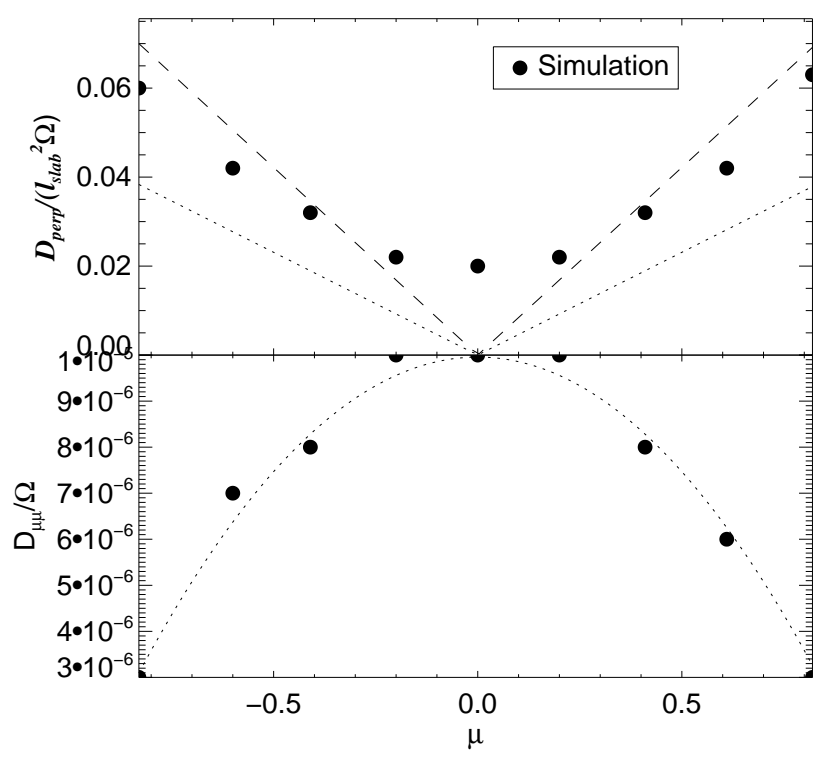

Figure 3. The same as Figure 2 except that $R_{L} / l_{\text {slab }}=10$

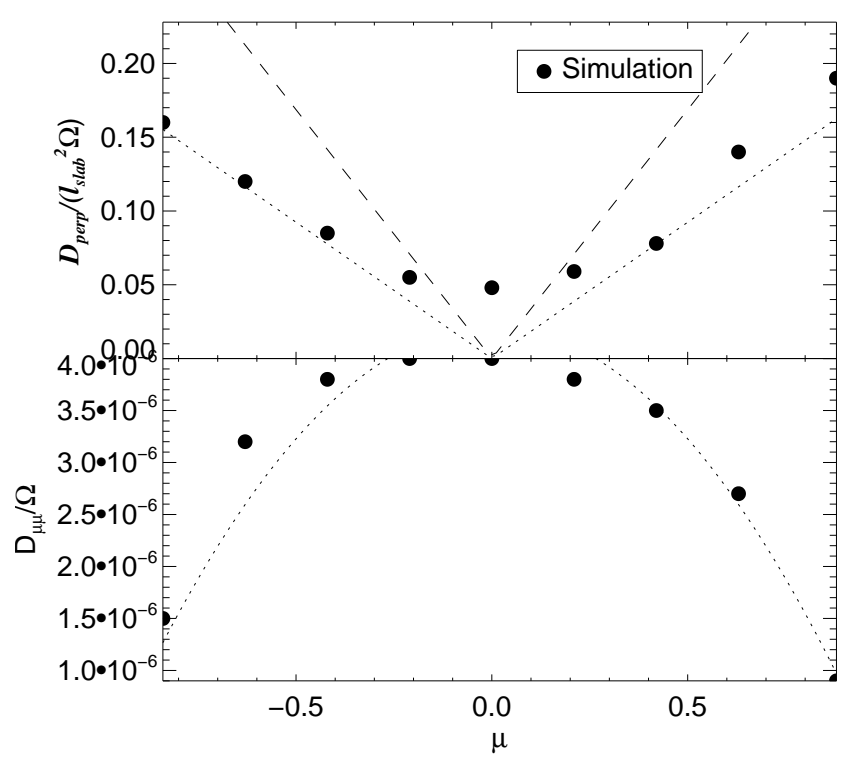

Figure 4 . The same as Figure 3 except that $R_{L} / l_{\text {slab }}=40$

\subsection{Parameters and Results for Run IV}

For the fourth run we set $R_{L} / l_{\text {slab }}=40$ and the results are shown in Figure 4. It is shown that when $|\mu|>0.1$ the simulation for $D_{\perp}(\mu)$ agree well with Equation (19) with $a^{2}=1 / 10$. But when $|\mu|<0.1$, the simulation results for 
$D_{\perp}(\mu)$ are much larger than the theoretical results from Equation (19). Similar as above, the simulation results for $D_{\mu \mu}(\mu)$ are almost isotropic.

\subsection{Parameters and Results for Run $V$}

To obtain Figure 5 we used $R_{L} / l_{\text {slab }}=100$. As long as $|\mu|>0.1$ the simulation results of $D_{\perp}(\mu)$ agree well with Equation (19) with $a^{2}=1 / 10$. But the fact that when $|\mu|>0$ the simulation results are smaller than the theoretical results with $a^{2}=1 / 10$ suggests a smaller value of $a^{2}$. In addition, when $|\mu|<0.1$, the simulation results of $D_{\perp}(\mu)$ is much larger than the theoretical results from Equation (19).

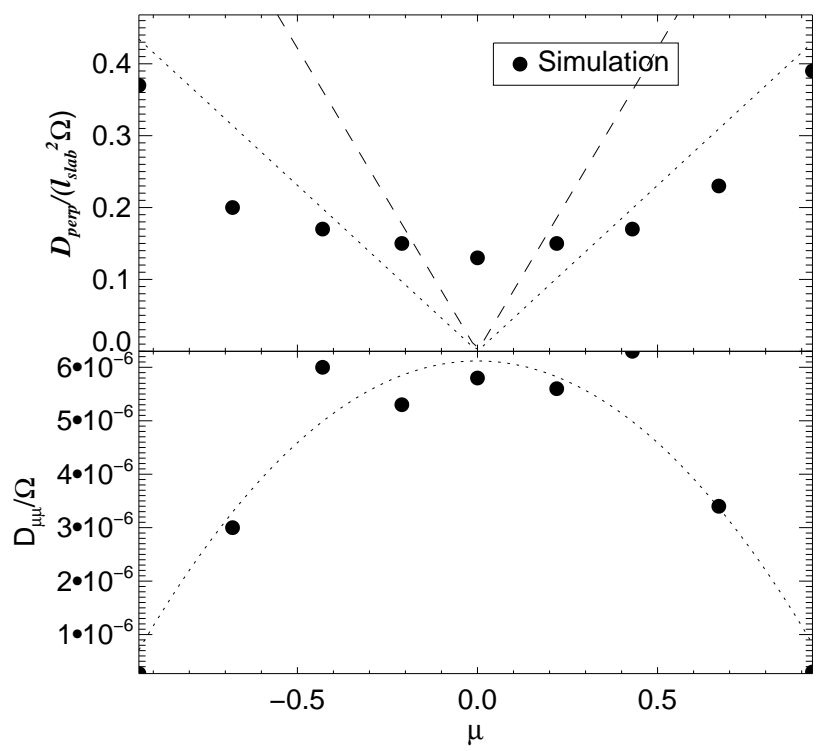

Figure 5. The same as Figure 4 except that $R_{L} / l_{\text {slab }}=100$

\subsection{Parameters and Results for Run VI}

Figure 6 is similar as Figure 5 except that we set a different ratio for the bendover scale, namely $l_{2 D}: l_{\text {slab }}=0.5$. We get similar results as from Figure 5 except that when $|\mu|>0.4$, the simulation results of $D_{\perp}(\mu)$ is much smaller than the theoretical results and $a^{2}$ should be much smaller than $1 / 10$.

From Figures 1-6 it is shown that $D_{\perp}$ is an even function of $\mu$ and it agrees well with Equation (19) with $a^{2}$ varying from $1 / 3$ to less than $1 / 10$. Furthermore, $D_{\mu \mu}$ can be assumed as an isotropic form (27) with a pitch-angle independent constant $D$ for the most cases. 


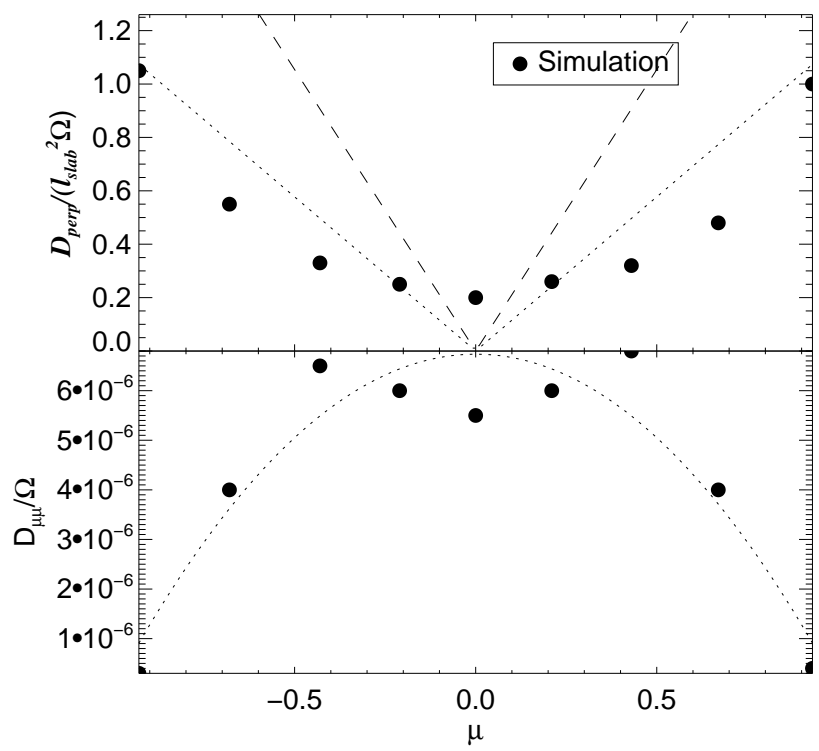

Figure 6. The same as Figure 5 except that $l_{2 D} / l_{\text {slab }}=0.5$

\subsection{Discussion}

In the six different runs we explored the pitch-angle dependence of the perpendicular Fokker-Planck coefficient $D_{\perp}(\mu)$. We also computed the pitch-angle scattering coefficient $D_{\mu \mu}(\mu)$. We compared our numerical results with the isotropic form (27) to complement the work done in Qin and Shalchi (2009). Furthermore, we compared the parameter $D_{\perp}(\mu)$ with the prediction made my the UNLT theory developed by Shalchi (2010). We performed our test-particle calculations for different values of the magnetic rigidity $R=R_{L} / l_{\text {slab }}$. As shown in Figures. 1-6, the parameter $D_{\mu \mu}(\mu)$ is always close to the isotropic form except for low rigidities $R=0.1$. For the perpendicular Fokker-Planck coefficient $D_{\perp}(\mu)$ we found results which are mostly directly proportional to $|\mu|$ in agreement with Equation (19). We also found that for the different parameter sets we have different values of the parameter $a^{2}$ which enters different theories for perpendicular transport.

The results found here basically confirm Equation (19) and therewith the UNLT theory for perpendicular diffusion. Therefore, these results can be used in different astrophysical applications such as simulations of solar modulation and diffusive shock acceleration.

\section{Summary and Conclusion}

In the present paper we have explored the pitch-angle dependent perpendicular diffusion coefficient $D_{\perp}(\mu)$. The latter parameter is also known as Fokker-Planck coefficient of perpendicular diffusion. In previous simulations the pitch-angle averaged perpendicular diffusion coefficient $\kappa_{\perp}$ has been computed (see, e.g., Michałek \& Ostrowski, 1996; Mace et al., 2000; Qin et al., 2002a; 2002b; Tautz, 2010; Tautz \& Shalchi, 2013). To obtain the pitch-angle dependent transport parameters $D_{\mu \mu}(\mu)$ and $D_{\perp}(\mu)$ can be important for different astrophysical applications. To know $D_{\mu \mu}(\mu)$ and $D_{\perp}(\mu)$ is required if the Cosmic Ray Fokker-Planck equation is solved numerically. Florinski et al. (2008) and Zuo et al. (2011), for instance, studied the termination shock acceleration of pickup ions with the Fokker-Planck equation. Danos et al. (2013) solved the two-dimensional Fokker-Planck equation numerically to investigate the effect of adiabatic focusing on the motion of energetic particles and Qin et al. (2006) studied the effect of pitch-angle dependent adiabatic cooling on the Solar Energetic Particles (SEPs) by numerically solving the Fokker-Planck equation.

In the present paper we focus on pitch-angle dependent perpendicular diffusion. We have performed different runs (see Table 1) and we compare our findings with analytical results. The difficulty of the pitch-angle dependent transport is that pitch-angle scattering itself is usually very strong and the parameter $\mu$ is changing rapidly within a short time (see Qin \& Shalchi, 2009 for more details). Therefore, we had to find parameter regimes where $\mu$ remains almost constant within a sufficiently long time. In such cases we were able to obtain $D_{\perp}$ as a function of $\mu$. The obtained results agree very well with the pitch-angle dependent field line random walk limit (19) and the 
prediction made by the UNLT theory of Shalchi (2010).

\section{Acknowledgments}

Gang Qin acknowledges support by NNSFC 41125016, NNSFC 41074125, CMA grant GYHY201106011, and the Specialized Research Fund for State Key Laboratories of China. Andreas Shalchi acknowledges support by the Natural Sciences and Engineering Research Council (NSERC) of Canada.

\section{References}

Bieber, J. W., Matthaeus, W. H., Smith, C. W., Wanner, W., Kallenrode, M.-B., \& Wibberenz, G. (1994). Proton and electron mean free paths: The Palmer consensus revisited. The Astrophysical Journal, 420, 294-306. http://dx.doi.org/10.1086/173559

Danos, R. J., Fiege, J. D., \& Shalchi, A. (2013). Numerical Analysis of the Fokker-Planck Equation with Adiabatic Focusing: Isotropic Pitch-angle Scattering. The Astrophysical Journal, $772, \quad 35$. http://dx.doi.org/10.1088/0004-637X/772/1/35

Florinski, V., Zank, G. P., \& le Roux, J. A. (2008). Acceleration at the termination shock: Anisotropies and spectra. Advances in Space Research, 41, 361. http://dx.doi.org/10.1016/j.asr.2006.12.026

Jokipii, J. R. (1966). Cosmic-Ray Propagation. I. Charged Particles in a Random Magnetic Field. The Astrophysical Journal, 146, 480. http://dx.doi.org/10.1086/148912

Lerche, I., \& Tautz, R. C. (2011a). Cosmic ray diffusion: Detailed investigation of a recent model. Physics of Plasmas, 18, 082305. http://dx.doi.org/10.1063/1.3625277

Lerche, I. \& Tautz, R. C. (2011b). Response to "Comment on 'Cosmic ray diffusion: Detailed investigation of a recent model’ " [Phys. Plasmas 18, 114701 (2011)]. Physics of Plasmas, 18, 114702. http://dx.doi.org/10.1063/1.3660288

Mace, R. L., Matthaeus, W. H., \& Bieber, J. W. (2000). Numerical Investigation of Perpendicular Diffusion of Charged Test Particles in Weak Magnetostatic Slab Turbulence. The Astrophysical Journal, 538, $192-202$. http://dx.doi.org/10.1086/309093

Matthaeus, W. H., Bieber, J. W., Ruffolo, D., Chuychai, P., \& Minnie, J. (2007). Spectral Properties and Length Scales of Two-dimensional Magnetic Field Models. The Astrophysical Journal, 667, 956-962. http://dx.doi.org/10.1086/520924

Matthaeus W. H., Goldstein M. L., \& Aaron R. D. (1990). Evidence for the presence of quasi-two-dimensional nearly incompressible fluctuations in the solar wind. Journal of Geophysical Research, 95, 20673-20683. http://dx.doi.org/10.1029/JA095iA12p20673

Matthaeus, W. H., Gray, P. C., Pontius, D. H., Jr., \& Bieber, J. W. (1995). Spatial Structure and Field-Line Diffusion in Transverse Magnetic Turbulence. Physical Review Letters, 75, 2136-2139. http://dx.doi.org/10.1103/PhysRevLett.75.2136

Matthaeus, W. H., Qin, G., Bieber, J. W., \& Zank, G. P. (2003). Nonlinear Collisionless Perpendicular Diffusion of Charged Particles. The Astrophysical Journal, 590, L53-L56. http://dx.doi.org/10.1086/376613

Michałek, G., \& Ostrowski, M. (1996). Cosmic ray momentum diffusion in the presence of nonlinear Alfvén waves. Nonlinear Processes in Geophysics, 3, 66-76. http://dx.doi.org/10.5194/npg-3-66-1996

Parker, E. N. (1965). The passage of energetic charged particles through interplanetary space. Planet. Space Sci., 13, 9-49. http://dx.doi.org/10.1016/0032-0633(65)90131-5

Pommois, P., Zimbardo, G., \& Veltri, P. (2005). Energetic particle transport in anisotropic magnetic turbulence. Advances in Space Research, 35, 647-652. http://dx.doi.org/10.1016/j.asr.2004.11.002

Pommois, P., Zimbardo, G., \& Veltri, P. (2007). Anomalous, non-Gaussian transport of charged particles in anisotropic magnetic turbulence. Physics of Plasmas, 14, 012311. http://dx.doi.org/10.1063/1.2434795

Qin, G., He, H.-Q., \& Zhang, M. (2011). An Effect of Perpendicular Diffusion on the Anisotropy of Solar Energetic Particles from Unconnected Sources. The Astrophysical Journal, $738,28$. http://dx.doi.org/10.1088/0004-637X/738/1/28

Qin, G., Matthaeus, W. H., \& Bieber, J. W. (2002a). Subdiffusive transport of charged particles perpendicular to the 
large scale magnetic field. Geophysical Research Letters, 29, 1048. http://dx.doi.org/10.1029/2001GL014035

Qin, G., Matthaeus, W. H., \& Bieber, J. W. (2002b). Perpendicular Transport of Charged Particles in Composite Model Turbulence: Recovery of Diffusion. The Astrophysical Journal, 578, L117-L120. http://dx.doi.org/10.1086/344687

Qin, G., \& Shalchi, A. (2009). Pitch-Angle Diffusion Coefficients of Charged Particles from Computer Simulations. The Astrophysical Journal, 707, 61-66. http://dx.doi.org/10.1088/0004-637X/707/1/61

Qin, G., \& Shalchi, A. (2012). Numerical investigation of the influence of large turbulence scales on the parallel and perpendicular transport of cosmic rays. Advances in Space Research, 49, 1643-1652. http://dx.doi.org/10.1016/j.asr.2012.02.035

Qin, G., Zhang, M., \& Dwyer, J. R. (2006). Effect of adiabatic cooling on the fitted parallel mean free path of solar energetic particles. Journal of Geophysical Research: Space Physics, 111, A08101.

Ruffolo, D., Chuychai, P., \& Matthaeus, W. H. (2006). Random Walk of Magnetic Field Lines in Nonaxisymmetric Turbulence. The Astrophysical Journal, 644, 971-980. http://dx.doi.org/10.1086/503625

Ruffolo, D., Chuychai, P., Wongpan, P., Minnie, J., Bieber, J. W., \& Matthaeus, W. H. (2008). Perpendicular Transport of Energetic Charged Particles in Nonaxisymmetric Two-Component Magnetic Turbulence. The Astro$\begin{array}{lll}\text { physical Journal, } & 686, & 1231-1244 .\end{array}$ http://dx.doi.org/10.1086/591493

Ruffolo, D., Matthaeus, W. H., \& Chuychai, P. (2004). Separation of Magnetic Field Lines in Two-Component Turbulence. The Astrophysical Journal, 614, 420-434. http://dx.doi.org/10.1086/423412

Ruffolo, D., Pianpanit, T., Matthaeus, W. H., \& Chuychai, P. (2012). Random Ballistic Interpretation of Nonlinear Guiding Center Theory. The Astrophysical Journal, 747, L34. http://dx.doi.org/10.1088/2041-8205/747/2/L34

Schlickeiser, R. (2002). Cosmic Ray Astrophysics. Berlin: Springer. http://dx.doi.org/10.1007/978-3-662-04814-6

Shalchi, A. (2006). Extended nonlinear guiding center theory of perpendicular diffusion. Astronomy and Astrophysics, 453, L43-L46. http://dx.doi.org/10.1051/0004-6361:20065465

Shalchi, A. (2009). Nonlinear Cosmic Ray Diffusion Theories, Astrophysics and Space Science Library (Vol. 362). Berlin: Springer. http://dx.doi.org/10.1007/978-3-642-00309-7

Shalchi, A. (2010). A Unified Particle Diffusion Theory for Cross-field Scattering: Subdiffusion, Recovery of Diffusion, and Diffusion in Three-dimensional Turbulence. The Astrophysical Journal, 720, L127-L130. http://dx.doi.org/10.1088/2041-8205/720/2/L127

Shalchi, A. (2011a). Magnetic Field Line Random Walk in Two-dimensional Turbulence: Markovian Diffusion versus Superdiffusion. Contributions to Plasma Physics, 51, 920-930. http://dx.doi.org/10.1002/ctpp.201100106

Shalchi, A. (2011b). Comment on "Cosmic ray diffusion: Detailed investigation of a recent model" [Phys. Plasmas 18, 082305 (2011)]. Physics of Plasmas, 18, 114701. http://dx.doi.org/10.1063/1.3660287

Shalchi, A. (2013). Simple Analytical Forms of the Perpendicular Diffusion Coefficient for Two-component Turbulence. I. Magnetostatic Turbulence. The Astrophysical Journal, 774, 7. http://dx.doi.org/10.1088/0004-637X/774/1/7

Shalchi, A., Bieber, J. W., \& Matthaeus, W. H. (2004). Analytic Forms of the Perpendicular Diffusion Coefficient in Magnetostatic Turbulence. The Astrophysical Journal, 604, 675-686. http://dx.doi.org/10.1086/382128

Shalchi, A. \& Weinhorst, B. (2009). Random walk of magnetic field lines: Subdiffusive, diffusive, and superdiffusive regimes. Advances in Space Research, 43, 1429-1435. http://dx.doi.org/10.1016/j.asr.2008.12.022

Tan, L. C., Reames, D. V., Ng, C. K., Saloniemi, O., \& Wang, L. (2009). Observational Evidence on the Presence of an Outer Reflecting Boundary in Solar Energetic Particle Events. The Astrophysical Journal, 701, 1753. http://dx.doi.org/10.1088/0004-637X/701/2/1753

Tautz, R. C. (2010). Simulation results on the influence of magneto-hydrodynamic waves on cosmic ray particles. Plasma Physics and Controlled Fusion, 52, 045016. http://dx.doi.org/10.1088/0741-3335/52/4/045016

Tautz, R. C., \& Shalchi, A. (2013). Simulated energetic particle transport in the interplanetary space: The Palmer 
consensus revisited. Journal of Geophysical Research: Space Physics, 118, 642-647. http://dx.doi.org/10.1002/jgra.50155

Teufel, A., \& Schlickeiser, R. (2003). Analytic calculation of the parallel mean free path of heliospheric cosmic rays. II. Dynamical magnetic slab turbulence and random sweeping slab turbulence with finite wave power at small wavenumbers. Astronomy and Astrophysics, 397, 15-25. http://dx.doi.org/10.1051/0004-6361:20021471

Zank, G. P., Li, G., Florinski, V., Matthaeus, W. H., Webb, G. M., \& le Roux, J. A. (2004). Perpendicular diffusion coefficient for charged particles of arbitrary energy. Journal of Geophysical Research: Space Physics, 109, A04107.

Zank, G. P., \& Matthaeus, W. H. (1993). Nearly incompressible fluids. II - Magnetohydrodynamics, turbulence, and waves. Physics of Fluids A, 5, 257-273. http://dx.doi.org/10.1063/1.858780

Zhang, M. (1999). A Markov Stochastic Process Theory of Cosmic-Ray Modulation. The Astrophysical Journal, 513, 409-420. http://dx.doi.org/10.1086/306857

Zimbardo, G., Pommois, P., \& Veltri, P. (2006). Superdiffusive and Subdiffusive Transport of Energetic Particles in Solar Wind Anisotropic Magnetic Turbulence. The Astrophysical Journal, 639, L91-L94. http://dx.doi.org/10.1086/502676

Zuo, P. B., Zhang, M., Gamayunov, K., Rassoul, H., \& Luo, X. (2011). Energy Spectrum of Energetic Particles Accelerated by Shock Waves: From Focused Transport to Diffusive Acceleration. The Astrophysical Journal, 738, 168. http://dx.doi.org/10.1088/0004-637X/738/2/168

\section{Copyrights}

Copyright for this article is retained by the author(s), with first publication rights granted to the journal.

This is an open-access article distributed under the terms and conditions of the Creative Commons Attribution license (http://creativecommons.org/licenses/by/3.0/). 